\title{
Plasma exchange and intravenous immunoglobulin improved symptoms in a subtype of obsessive compulsive disorder and tic disorders
}

Perlmutter SJ, Leitman SF, Garvey MA, et al. Therapeutic plasma exchange and intravenous immunoglobulin for obsessive-compulsive disorder and tic disorders in childhood. Lancet 1999 Oct 2;354:1153-8.

QUESTION: In children with infection triggered exacerbations of obsessive compulsive disorder (OCD) or tic disorders, how effective are plasma exchange and intravenous immunoglobulin (IVIG) in improving neuropsychiatric symptoms?

Source of funding: $n o$ external funding: Some of the IVIG supplied by Baxter-Hyland corporation.

In children with infection triggered exacerbations of obsessive compulsive disorder or tic disorders, plasma exchange and intravenous immunoglobulin prompted rapid and sustained improvements in neuropsychiatric symptoms.

*See glossary.

†Information provided by author.

For correspondence: $D r$ SE Swedo, 10 Center Drive-MSC 1255,

Bethesda, $M D$ 20892-1255, USA

\section{Setting}

US National Institute of Mental Health (NIMH) inpatient ward and outpatient clinic.

\section{Patients}

30 children who were 5 -14 years of age (mean age $10 \mathrm{y}$, $63 \%$ boys) and met the DSM-III diagnosis for OCD, a tic disorder, or both. Other inclusion criteria were onset of neuropsychiatric signs and symptoms before puberty, history of sudden onset of signs and symptoms or an episodic course with abrupt exacerbations and periods of remission, evidence of an association between streptococcal infection and onset of signs and symptoms, and current exacerbation causing substantial distress and interfering with the child's social functioning in $\geqslant 2$ of home, school, or social relations. Exclusion criteria were history of Sydenham's chorea or rheumatic fever, autism, schizophrenia or other psychotic disorder, other neurological disorder, or medical illness. Follow up was $97 \%$ at 1 month and $90 \%$ at 1 year.

\section{Intervention}

Patients were allocated to plasma exchange $(n=10)$, IVIG, $1 \mathrm{~g} / \mathrm{kg}$ of body weight for 2 days $(\mathrm{n}=10)$, or placebo $(n=10)$. In patients allocated to plasma exchange, 1 plasma volume ( $45 \mathrm{ml} / \mathrm{kg}$ of body weight) was exchanged 5-6 times over a course of 10-12 days.

\section{Main outcome measures}

Change from baseline in obsessive compulsive symptoms, anxiety, depression, emotional lability, global functioning, and global severity.

\section{Main results}

At 1 month, patients in IVIG and plasma exchange groups each had greater improvement from baseline than did patients in the placebo group for obsessive compulsive symptoms (Yale-Brown Obsessive Compulsive Scale); anxiety, depression, and emotional lability (NIMH scales); global function (NIMH global scale); and global severity (Clinical Global Impression Severity scale) (table). Tic severity was improved from baseline in the plasma exchange group but not in the IVIG group compared with placebo. Improvements from baseline were maintained at 1 year.
Intravenous immunoglobulin (IVIG) or plasma exchange v placebo for obsessive compulsive disorder and tic disorder at 1 month

Outcomes Points improvement in score from baseline (\% change)

\begin{tabular}{llll} 
& IVIG & Plasma exchange & Placebo \\
\hline Obsessive compulsive symptoms & $12(45 \%)$ & $13(58 \%)$ & $0.9(3 \%)$ \\
\hline Tics & $1.3(19 \%) \ddagger$ & $10.7(58 \%)$ & $1.3(12 \%)$ \\
\hline Anxiety & $2.1(31 \%)$ & $3.0(47 \%)$ & $0.2(3 \%)$ \\
\hline Depression & $1.4(26 \%)$ & $2.3(44 \%)$ & $-0.1(2 \%)$ \\
\hline Emotional lability & $1.8(29 \%)$ & $2.2(35 \%)$ & $-0.1(2 \%)$ \\
\hline Global function & $2.9(33 \%)$ & $2.8(35 \%)$ & $0(0 \%)$ \\
\hline Global severity & $1.3(26 \%)$ & $1.8(36 \%)$ & $0(1 \%)$ \\
\hline
\end{tabular}

fNot significant for comparison with placebo.

\section{COMMENTARY}

Swedo $e$ al have been studying the role of post streptococcal immunity in neuropsychiatric disorders for several years and have defined the concept of paediatric autoimmune neuropsychiatric disorder associated with streptococcal infection (PANDAS). ${ }^{1}$ This study by Perlmutter $e t$ al reports robust improvement in OCD symptoms with both IVIG and plasma exchange and essentially no change in symptoms with placebo intervention. Plasma exchange had a similarly pronounced beneficial effect on tic severity whereas IVIG and placebo treatments showed only minor improvement. One must keep in mind, however, that the baseline severity of tic disorders was lower in the IVIG and placebo groups than in the plasma exchange group, hence no conclusions can be drawn about the efficacy of IVIG in treating tics. The effects of the experimental treatment were sustained at up to 1 year except in 3 patients who had a history of new streptococcal exposure confirmed by anti-streptococcal titres and who required re-treatment. It is notable that re-infection occurred despite oral penicillin prophylaxis.

Although both treatments reduced the severity of OCD symptoms by about $50 \%-60 \%$, they do not provide a cure. 11 of 13 children who started the study on medication continued to require the medication albeit sometimes at a reduced dose.

The results of this study offer support for the role of immune mechanisms in the aetiology and treatment of neuropsychiatric symptoms and provide intriguing leads for further investigations. It is also clear, however, that these results are based on a specific, highly selected population of children with $\mathrm{OCD}$ and/or tic disorder who met diagnostic criteria for PANDAS. It remains to be shown for which patients immunomodulatory therapy may be effective. Much more work is required before immunomodulatory treatments can be used routinely outside research protocols.

Paul Sandor, MD, FRCPC Toronto Western Hospital,

Toronto, Ontario, Canada

Swedo SE, Leonard HL, Garvey M, et al. Pediatric autoimmune neuropsychiatric disorders associated with streptococcal infections: clinical description of the first 50 cases. Am J Psychiatry 1998;155:264-71. 